\title{
Role of block copolymer surfactant on the pore formation in methylsilsesquioxane aerogel systems
}

\section{AUTHOR(S):}

Kurahashi, Masayuki; Kanamori, Kazuyoshi; Takeda, Kazuyuki; Kaji, Hironori; Nakanishi, Kazuki

\section{CITATION:}

Kurahashi, Masayuki ...[et al]. Role of block copolymer surfactant on the pore formation in methylsilsesquioxane aerogel systems. RSC Advances 2012, 2(18): 7166-7173

ISSUE DATE:

2012-07

URL:

http://hdl.handle.net/2433/174711

\section{RIGHT:}

(C) 2012 Royal Society of Chemistry; This is not the published version.

Please cite only the published version.; この論文は出版社版でありませ ん。引用の際には出版社版をご確認ご利用ください。 


\title{
Role of block copolymer surfactant on the pore formation in methylsilsesquioxane aerogel systems
}

Masayuki Kurahashi, ${ }^{a}$ Kazuyoshi Kanamori, ${ }^{a}{ }^{a}$ Kazuyuki Takeda, ${ }^{a}$ Hironori Kaji ${ }^{b}$ and Kazuki Nakanishi ${ }^{a}$

a Department of Chemistry, Graduate School of Science, Kyoto University, Kitashirakawa, Sakyo-ku, Kyoto 606-8502, Japan. Fax: +81-75-753-7673; Tel: +81-75-753-7673

b Institute for Chemical Research, Kyoto University, Uji, Kyoto 611-0011, Japan. Fax: +81-774-38-3149; Tel: +81-774-38-3149

*Corresponding author

E-mail: kanamori@kuchem.kyoto-u.ac.jp

\begin{abstract}
$\underline{\text { Abstract }}$
Transparent and low-density methylsilsesquioxane (MSQ, $\mathrm{CH}_{3} \mathrm{SiO}_{1.5}$ ) aerogels can be obtained solely from methyltrimethoxysilane (MTMS) by a one-pot two-step process under the co-presence of surfactant. In the present study, we have systematically investigated effects of molecular structure of triblock copolymer-type nonionic surfactants PEO- $b$-PPO- $b$-PEO (PEO and PPO denote poly(ethylene oxide) and poly(propylene oxide) units, respectively) on the properties of resultant MSQ aerogels. Macroscopic phase separation of hydrophobic MSQ networks from polar solvent occurs when no surfactant is employed, which results in macroporous opaque aerogels. In contrast, a co-presence of appropriate surfactant effectively suppresses the phase separation and yields transparent aerogels after supercritical drying. By employing various surfactants having different molecular weight and PO/EO ratio, the mechanism of suppression of phase separation or pore formation is discussed in detail. In-situ ${ }^{1} \mathrm{H}$ NMR suggests that the PO units of surfactant interact with the hydrophobic MSQ network enriched with methyl groups and make the MSQ network hydrophilic by extending EO chains toward aqueous solvent in the late phase of gelation, until which hydrogen bonding dominates between $\mathrm{Si}-\mathrm{OH}$ groups of polymerizing MSQ and the ether oxygens of EO unit. Through the comprehensive understanding of the role of surfactant, the strategy for rational design of MSQ aerogels materials has become developable.
\end{abstract}

\section{Introduction}

Rational and controlled design of porous materials prepared in liquid media requires 
sufficient understanding of chemical reactions and interactions of components through the pore formation process in solution. For instance, typical mesoporous materials are prepared under the controlled hydrolysis-polycondensation reaction of metal alkoxides with the aid of co-assembly of surfactant. ${ }^{1}$ Attractive interaction between polymerizing inorganic species and surfactant through electrostatic interaction or hydrogen bonding is essential for the formation of mesopores with ordered periodicity and tunable properties in these materials. $^{1-4}$

Amongst various porous materials prepared in liquid media such as sol-gel, aerogel is another unique representative with very high porosity $(\sim 90 \%)$ and is gaining increasing attention due to the unique features, a wide variety in chemical compositions, and potential applications to energy-related materials as detailed below. ${ }^{5-7}$ While aerogels in various chemical compositions, such as inorganic oxides, hybrids, organic polymers, and carbons, have been reported since the aerogel was first prepared in more than 80 years ago, ${ }^{8}$ silica aerogels remain in the heart of most active research. The silica aerogels possess a lot of interesting properties such as high optical transparency, high specific surface area, low acoustic/thermal conductivity, low refractive index, and low dielectric constant. Extended applications therefore have been expected or established for such as Cherenkov counters, low- $k$ materials, cosmic dust collectors, catalyst supports, electrodes, and transparent thermal insulators. ${ }^{5-7,9,10}$ In particular, for more efficient use of energy, the demand for aerogel thermal insulators with lower thermal conductivity than the conventional ones such as glass wools and polymer foams is rapidly increasing due to the urgent global energy-related issues.

The unique features are derived from the distinct porous structures consisting of $\sim 10$ $\mathrm{nm}$ particles forming a "string of pearls"-type structure represented by $30-50 \mathrm{~nm}$ pores. However, the fatal problem associated with the lack of mechanical durability, which is originated from the abovementioned structural features, still remains. To resolve this problem, much work has been done; extensive aging in a mother solution, in a monomer solution, or in water, ${ }^{11-13}$ hybridization with organic polymers, ${ }^{14-16}$ etc. Organic-inorganic hybridization using organotrialkoxysilanes such as methyltrimethoxysilane (MTMS) as the single or co-precursor with tetraalkoxysilane, is a promising facile way to obtain mechanically durable and flexible aerogels and xerogels. ${ }^{17-19}$ These hybrid siloxane materials (silicone resins in other words) give flexible networks derived from inorganic frameworks with reduced crosslinking density and integrated organic moiety. However, 
only turbid aerogels and xerogels had been reported with the increasing ratio of organotrialkoxysilane due to the undesirable cyclization reactions and macroscopic phase separation from polar solvent. Our group for the first time synthesized transparent aerogels from MTMS as the single precursor by using surfactant to suppress the phase separation of hydrophobic MTMS-derived condensates, and urea as an in-situ base-releasing catalyst to homogeneously increase the solution $\mathrm{pH}$ to promote polycondensation. ${ }^{20-24}$ Two kinds of surfactant, cationic $n$-hexadecyltrimethylammonium salts (CTAB for bromide and CTAC for chloride) and nonionic poly(ethylene oxide)-block-poly(propylene oxide)-block-poly(ethylene oxide) (PEO- $b$-PPO- $b$-PEO) triblock copolymer (Pluronic F127: $\mathrm{EO}_{106} \mathrm{PO}_{70} \mathrm{EO}_{106}$ ) are found to effectively suppress the phase separation, leading to transparent methylsilsesquioxane (MSQ, $\mathrm{CH}_{3} \mathrm{SiO}_{1.5}$ ) aerogels. Owing to the flexibility and high strength against compressive stress in these MSQ aerogels, low-density and transparent MSQ xerogels can also be obtained by simple evaporative drying under ambient conditions, which significantly reduces the production cost and extended applications are highly expected.

For a perspective and rational design of these scientifically and technologically important materials, it is indispensable to understand the role of surfactant in the course of gelation and pore formation. In the case of tetraalkoxysilane such as tetramethoxysilane (TMOS), CTAB is known to interact with the silica domain by settling polar head groups toward silica, ${ }^{25}$ while PEO- $b$-PPO- $b$-PEO type triblock copolymers (typically P123 and F127) make hydrogen bonding between silanol groups ( $\mathrm{Si}-\mathrm{OH})$ and the ether oxygens of EO units. ${ }^{26}$ In contrast, since MTMS possesses hydrophobic organic groups $\left(-\mathrm{CH}_{3}\right)$, the MSQ condensates are basically more hydrophobic compared to TMOS-derived silica gels with abundant silanol groups. In addition, it is expected that the interaction mechanism of MTMS and surfactant are more complicated because the hydrolyzed monomers and oligomers possess a lot of silanol groups but increase hydrophobicity with the progress of polycondensation, which proceeds with the consumption of silanol groups. In the case of block copolymers, in particular, the suppression mechanism of phase separation, or interaction between MSQ condensates and surfactant, has been still unclear. In this study, we prepared aerogels from MTMS with a variety of triblock type surfactants (Pluronic F127, Synperonic F108, Pluronic F68, Synperonic P105, Pluronic L35 and Pluronic P123, properties listed in Table 1), ${ }^{27,28}$ and systematically investigated the changes in the properties of the obtained aerogels. Also, in-situ ${ }^{1} \mathrm{H}$ NMR is employed to obtain 
time-resolved information on each chemical component during polycondensation. The goal of this work is to clarify the interaction mechanism between MSQ and the nonionic surfactants during the condensation reaction for a better design of low-density and transparent MSQ aerogels and xerogels materials.

Table 1 Physical properties of triblock copolymers employed in this study

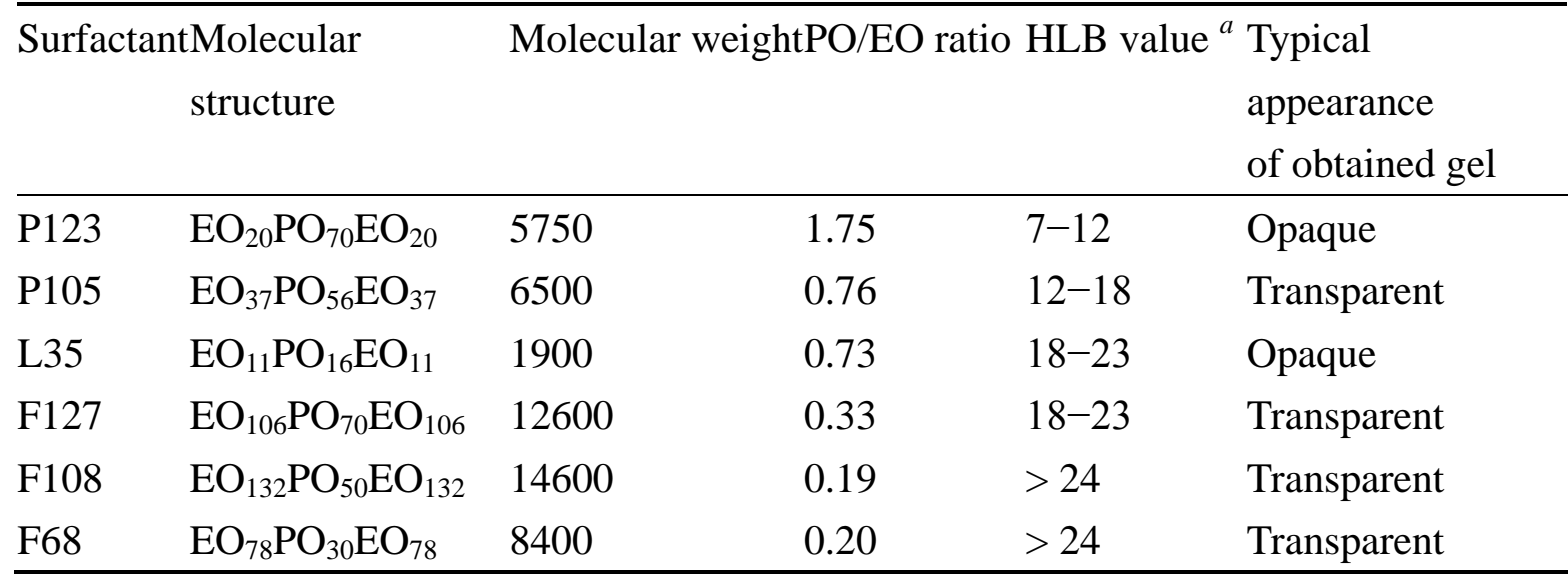

${ }^{a}$ Hydrophile-Lipophile Balance ${ }^{27}$

\section{Experimental}

Materials

Acetic acid was purchased from Kishida Chemical Ltd. (Japan). Distilled water and urea were from Hayashi Pure Chemical Ltd. (Japan). All of triblock copolymer type surfactants (Pluronic F127, Synperonic F108, Pluronic F68, Synperonic P105, Pluronic L35 and Pluronic P123) and methytrimethoxylsilane (MTMS, $98 \%$ ) were from Sigma-Aldrich Co. (USA). All reagents were used as received.

\section{Preparation of aerogels}

In a glass tube, $10 \mathrm{~mL}$ of $5 \mathrm{mM}$ aqueous acetic acid, a given amount of surfactant and $3.0 \mathrm{~g}$ of urea were mixed. Stirred the solution until the sol became homogeneous, and then 5 $\mathrm{mL}$ of MTMS was added into the vial under vigorous stirring. After $30 \mathrm{~min}$ stirring at room temperature for hydrolysis, the sol was transferred into a polystyrene mold for gelation. Gelation and aging for $4 \mathrm{~d}$ in $60{ }^{\circ} \mathrm{C}$ oven were followed by washing with methanol and solvent exchange with 2-propanol at $60{ }^{\circ} \mathrm{C}$ each for three times. Finally, $\mathrm{CO}_{2}$ supercritical drying at $80{ }^{\circ} \mathrm{C}$ under $14 \mathrm{MPa}$ for $10 \mathrm{~h}$ was conducted to obtain dried 
aerogel samples.

\section{Characterization}

Bulk density was calculated from the weight/volume ratio of the aerogel samples. Linear shrinkage was calculated from the ratio of the dimension of the dried samples to that of the mold used for gelation. For light transmittance measurement, UV-VIS spectrometer V-670 (JASCO Co., Japan) equipped with an integrating sphere ISN-723 was used, and direct-hemispherical transmittance at $550 \mathrm{~nm}$ was normalized to the value of $10 \mathrm{~mm}$ thickness by the Lambert-Beer equation. Microstructure of the samples was observed with a field-emission scanning electron microscope (FE-SEM, JSM-6700F, JEOL Ltd., Japan) in the nanometer range and with SEM (JSM-6060S, JEOL Ltd., Japan) in the micrometer range. Thermal analysis was carried out with a thermogravimetry-differential thermal analysis system (TG-DTA) (Thermoplus TG 8120, Rigaku Corp., Japan) at a heating rate of $5^{\circ} \mathrm{C} \min ^{-1}$ under an air flow.

\section{NMR Experiments}

Liquid-state ${ }^{1} \mathrm{H}$ NMR was performed on a Bruker Avance III 600US Plus operating at frequencies of $600.13 \mathrm{MHz}$, and the chemical shift was referenced to ${ }^{1} \mathrm{H}$ of sodium 3-(trimethylsilyl)propionate-2,2,3,3- $\mathrm{d}_{4}\left(\mathrm{TSP}-\mathrm{d}_{4}\right)$. To prepare the samples, deuterium oxide (99.990\%, Sigma-Aldrich Co., USA) was used instead of water to avoid spectrum overlap. The hydrolyzed solution was placed in a probe fixed at $60{ }^{\circ} \mathrm{C}$, and a series of the spectrum was recorded at predetermined time intervals.

Solid-state ${ }^{29} \mathrm{Si}$ CP/MAS NMR experiments were performed on an OPENCORE NMR at $59.50 \mathrm{MHz}{ }^{29,30}$ The chemical shifts were referenced to the silicon in tetramethylsilane. The contact time for the cross polarization was fixed at $10 \mathrm{~ms}$ and the rate of sample spinning was set to $8.7 \mathrm{kHz}$. The sample was dried at $60{ }^{\circ} \mathrm{C}$ under ambient pressure without washing, followed by drying under vacuum at $60{ }^{\circ} \mathrm{C}$ before the measurement to completely remove water inside the pores.

\section{Results and discussion}

Before going into discussion on the effects of surfactants, we briefly introduce the notation of surfactants. Following the nomenclature rule of the original manufacturer (BASF, Germany), the letter before their number in the names shows types of their physical state at 
room temperature; liquid $(\mathrm{L})$, paste $(\mathrm{P})$ or flake $(\mathrm{F})$. The first one or two digit(s) are related to their molecular weight of PO blocks in each molecule as shown in the vertical axis in Fig. 1. The last digit shows a tenth of weight \% of EO content in each molecule. For example, F108 and F68 are both in flake, and have the same digit " 8 " at the end of their name, which means both of them have $80 \mathrm{wt}$. \% of ethylene oxide in each molecule with longer PO units for F108.

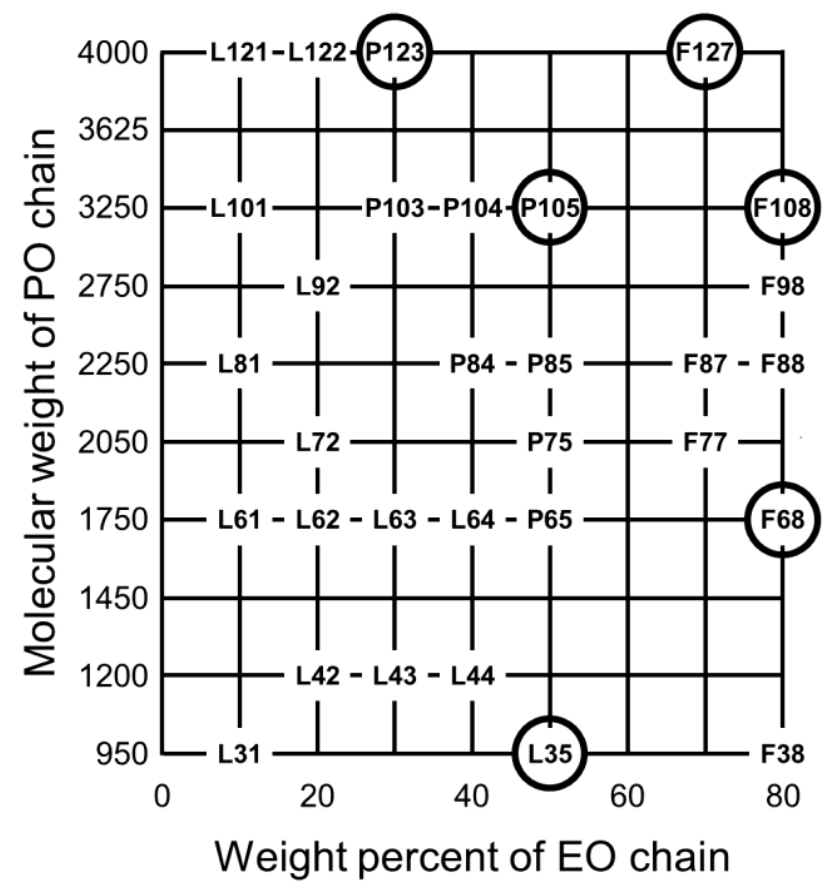

Fig. 1 The Pluronic grid. ${ }^{26,27}$ Surfactants used in this study are indicated by the circles.

\section{Changes by concentration of surfactant F108}

While only opaque monolithic gels which consist of particle aggregates in micrometer size are obtained without any surfactant as the result of phase separation of MSQ networks from aqueous solution, ${ }^{23}$ it is already known that appropriate triblock copolymer surfactant effectively suppresses the phase separation. However, it has not been investigated how the porous morphology and molecular-level structures in the MSQ gels change by the co-presence of surfactants with different EO/PO ratios and molecular weight in detail. Fig. 2 shows the changes in light transmittance, bulk density and total shrinkage during both aging and supercritical drying processes in the aerogels prepared with F108. Based on the weight of F108, the variation tendency of properties can be divided into three different 
regions.

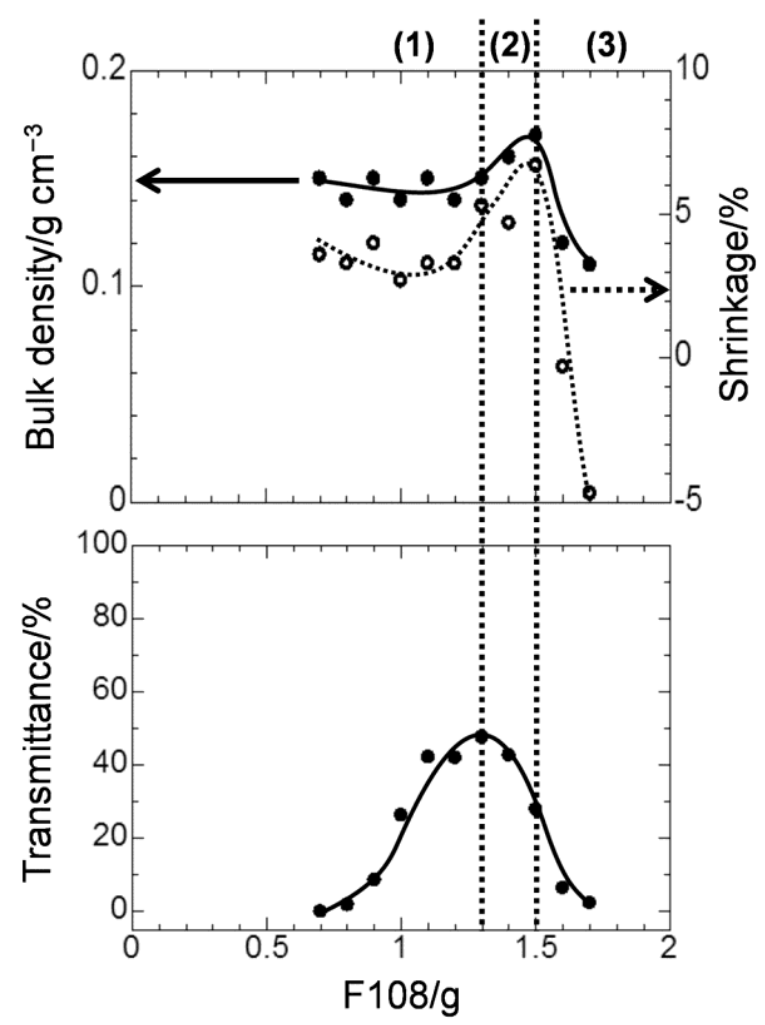

Fig. 2 Changes in light transmittance, bulk density and shrinkage with the amount of F108.

In region (1), light transmittance increases with the increasing amount of surfactant and the size of porous structure becomes finer from tens of micrometers to less than a hundred nanometers as shown in Fig. 3a-c. Without any surfactant, as described above, only opaque macroporous gels were obtained due to the micrometer-scaled phase separation of hydrophobic condensates from the polar aqueous solvent. With increasing amount of surfactant, domain size becomes smaller and the visible light scattering is reduced owing to the suppression of phase separation. 

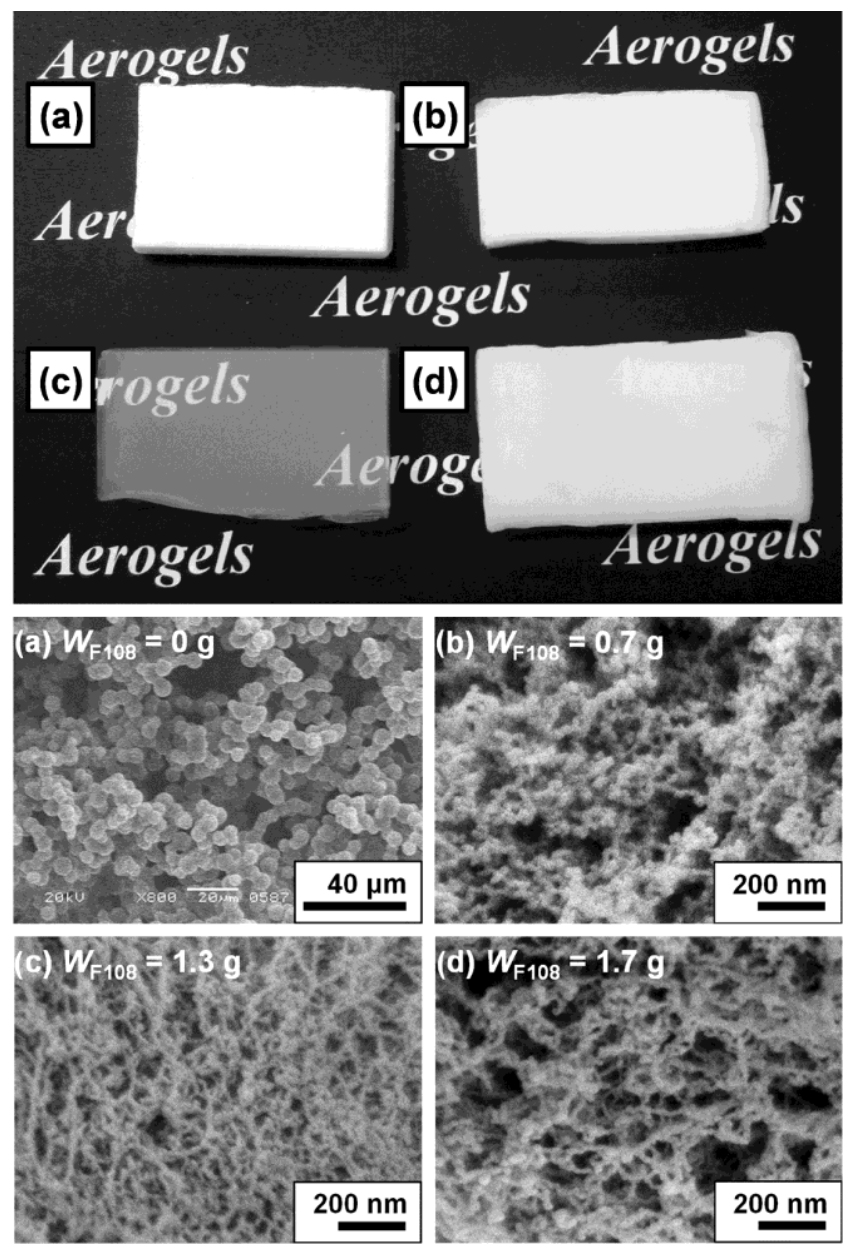

Fig. 3 Appearances and porous structures of the samples prepared with different amounts of F108; (a) $0 \mathrm{~g}$, (b) $0.7 \mathrm{~g}$, (c) $1.3 \mathrm{~g}$ and (d) $1.7 \mathrm{~g}$

In region (2), transparency decreases and bulk density increases. In this region, shrinkage of the gel samples is higher, which may cause inhomogeneity and transparency decreases. As discussed later with the ${ }^{29} \mathrm{Si}$ solid-state NMR data, crosslinking density increases with increasing amount of surfactant. However, the connection between colloidal MSQ becomes increasingly loose presumably by the increasing entanglement with surfactant molecules in the course of gelation. As a result, the pore skeletons shown in Fig. 3 become softer and deformable, which contributes to the higher shrinkage in this region.

In region (3), the excess amount of F108 offers more entanglement with the condensates, making the networks increasingly loose. In other words, the looser networks 
than those in region b) extensively increase the deformability and the gels become to exhibit swelling by taking solvent inside the gel body during the washing and solvent-exchanging steps with alcohols. In our previous swelling study on the lightly crosslinked MSQ gels, the MSQ network showed low polarity (solubility parameter less than $10 \mathrm{cal}^{1 / 2} \mathrm{~cm}^{-3 / 2}$ ). The swelling is therefore deduced to occur by the supercritical $\mathrm{CO}_{2}$ during drying as well, and the resultant aerogel shown in Fig. 3d may reflect the swollen state. The resultant shrinkage and bulk density consequently become lower and the inhomogeneous pore structure leads to lower light transmittance. In the case of other surfactants which gives transparent gels (F127, F68 and P105), similar behaviors are also confirmed (Fig. 4). In the case of P105, which have the smallest EO content in these four surfactants, region c) cannot be observed (i.e. no swelling observed), presumably due to the lower molecular weight. Note here that a starting solution with more than $3.2 \mathrm{~g}$ of P105 was not suitable for gelation because of a significant increase of viscosity.
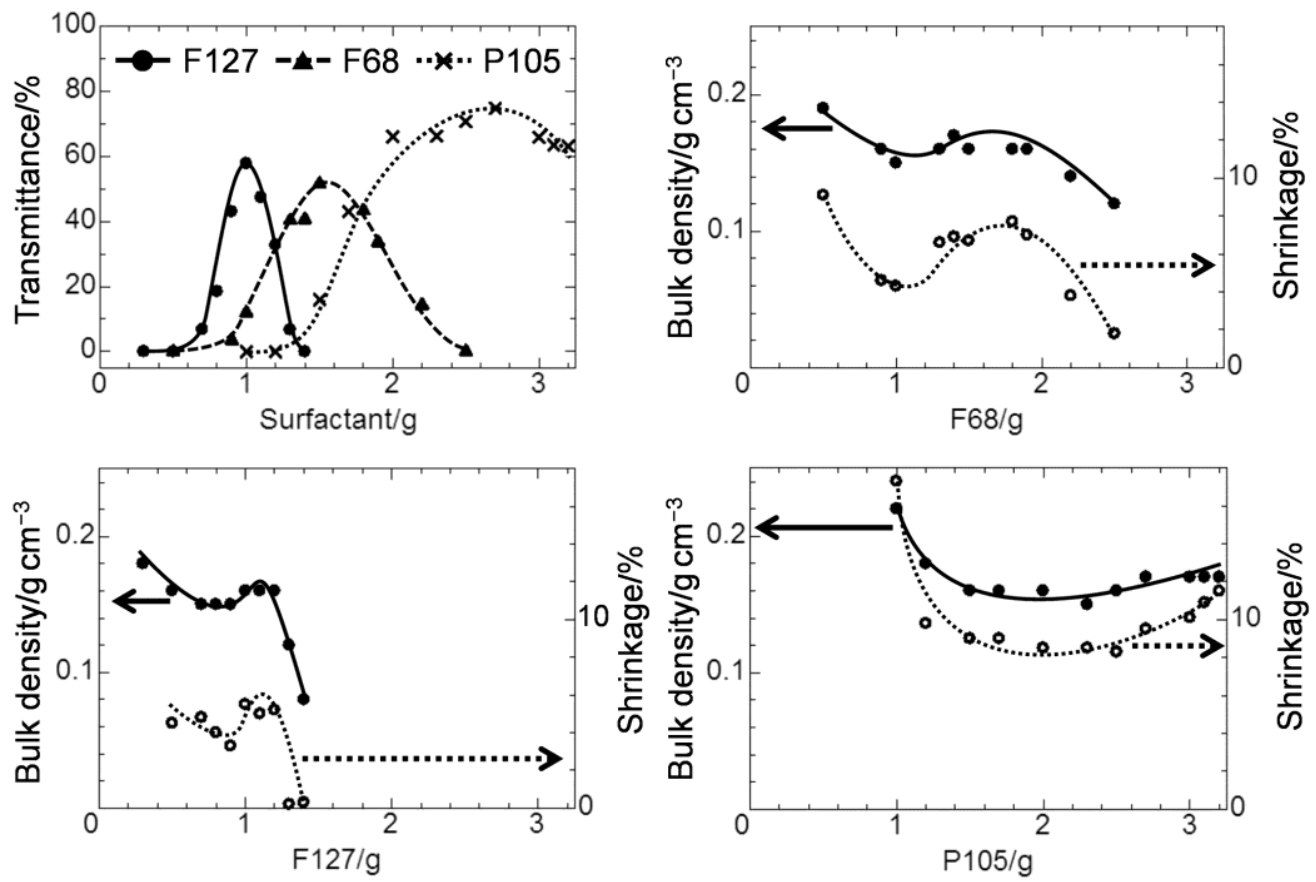

Fig. 4 Changes in light transmittance, bulk density and shrinkage in the systems incorporated with F127, F68 and P105.

\section{Effect of molecular weight}

Let us start the discussion on effect of molecular weight of surfactant by comparing the pair 
of F108 and F68, both of which gave transparent aerogels, and then the other pair of P105 and L35. The surfactant F68 has the lower molecular weight with 80 wt. \% of EO chains (the same EO content as F108), and suppressed phase separation of MSQ effectively, leading to the aerogels with almost the same transparency as those prepared with F108. Microstructures in these two systems have no distinct differences as shown in Figs. 3c and 5a for the highest light transmittance samples in each system. Only the difference between these two systems is the amount of surfactant to attain the highest light transmittance (1.3 g for F108 and $1.5 \mathrm{~g}$ for F68), i.e. F68 with lower molecular weight needs larger amount to suppress the phase separation to the same extent. With $1.3 \mathrm{~g}$ of F68, the phase separation is not well-suppressed (the lower light transmittance) nevertheless the addition of the same weight of polymers directly means that the same numbers of EO and PO units are present in the solution. As discussed later in detail, interactions between polymeric surfactant and MSQ networks play the key factor in the suppression of phase separation; the MSQ networks are rendered hydrophilic through the interactions in the course of gelation. It is well known that as molecular weight increases, the number of available sites for adsorption increases. When adsorbed on a solid surface through a specific interaction, higher molecular weight polymers generally show the higher stability in a given system than lower molecular weight ones with the same composition, because the probability of desorption of the longer polymers is lower, while the shorter ones tend to receive the higher hydrodynamic driving force for desorption. ${ }^{31,32}$

This tendency becomes more distinct when comparing the surfactants P105 and L35, both of which contain 50 wt. \% EO units but the molecular weights are significantly different (6500 for P105 and 1900 for L35). The surfactant P105 most effectively suppressed the phase separation in the present study and gave the most transparent aerogel ( $T=75 \%$, Fig. 4), while only opaque gels were obtained from L35. Microstructural morphology is quite different between these two (Fig. 5b and c). Judging from the microstructure in L35 (5.0 g) consisting of larger particles, the suppression of phase separation appears insufficient. Since the chain length of L35 is too short, the number of adsorption sites to MSQ networks is significantly smaller. This means that the L35 polymers therefore cannot effectively interact with MSQ networks, which keeps the MSQ networks hydrophobic in the polar solvent. Inhomogeneous pore structure with the coarser structure can be seen by this reason. On the other hand, the sample from P105 system $(2.7 \mathrm{~g})$ consists of finer particles with the more uniform pore structure, and large 
aggregates and inhomogeneity cannot be observed.
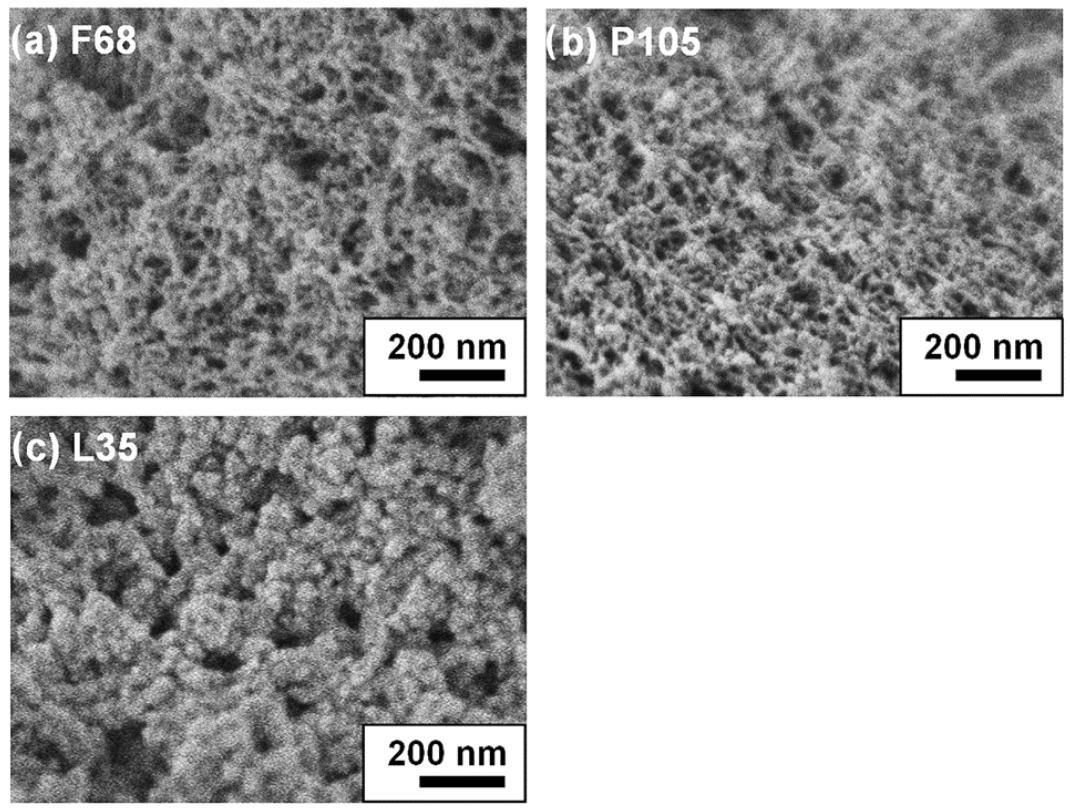

Fig. 5 Porous structures of aerogels prepared with (a) F68 (1.5 g), (b) P105 (2.7 g) and (c) L35 (5.0 g).

\section{Influence of $\mathrm{PO} / \mathrm{EO}$ unit ratio}

Next we compare F108 and P105, both of which have the same amount of PO units ( 50) but the different EO chain lengths; 264 EO units for F108 and 74 for P105. The amount of surfactant needed to obtain the most transparent aerogels in P105 system $(2.7 \mathrm{~g})$ was more than twice the amount in F108 system $(1.3 \mathrm{~g})$ as shown in Figs. 2 and 4 . Also, the maximum light transmittance is higher in P105 system $(T=75 \%)$ compared to F68 system $(T=52 \%)$. This behavior can be explained by the ratio of $\mathrm{PO}$ and $\mathrm{EO}$ units, hydrophobicity or HLB in other words, of the surfactants listed in Table 1. In P105 system, PO/EO ratio is higher than F108, F68 and F127 systems, and it can be confirmed that the more hydrophobic surfactant allows MSQ networks to become more hydrophilic except for L35 and P123 (vide infra). It therefore can be proposed that hydrophobic interaction between PO and MSQ networks is dominant ${ }^{33-35}$ when the polycondensation enough proceeds and MSQ networks consume hydrophilic silanol groups. Shorter EO chains in P105 also take advantage for liberating from MSQ networks after silanol groups

are consumed. The liberated EO groups are exposed toward the polar solvent ${ }^{34,36}$ and effectively contribute to suppress the phase separation. 
When comparing surfactants having 70 PO units in each molecule (F127 and P123), the properties of obtained samples are quite different from the previous examples with 50 PO units; P123 did not give any transparent gel. All of the gels prepared with P123 were opaque and their structure was in the micrometer order, while gels prepared with F127 were transparent having nanometer-scale structures (Fig. 6). From the above discussion, one might anticipate that P123 with higher hydrophobicity (Table 1) most effectively suppresses phase separation. However, they distribute inside (not at the interface of) the MSQ networks $^{37,38}$ and do not contribute to the suppression of phase separation due to the too high hydrophobicity.
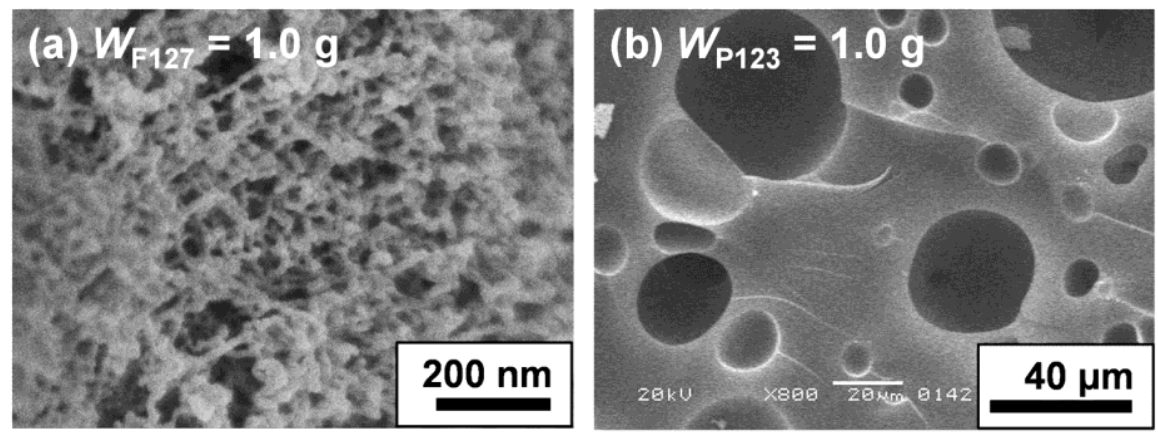

Fig. 6 Porous structures of aerogels prepared with F127 (a) and P123 (b).

To confirm the distribution of P123, we carried out the TG-DTA experiment on the macroscopically phase-separated (separated into precipitate and supernatant) and evaporatively-dried sample prepared with P123. In Fig. 7, TG-DTA curves for the gel and solvent phases together with MSQ gels prepared with no surfactant are presented. The weight losses observed at the temperature ranges of $140-185{ }^{\circ} \mathrm{C}$ and $205-305{ }^{\circ} \mathrm{C}$ are attributed to the decomposition of remaining urea, ${ }^{39}$ which are overlapped with several thermal events such as additional condensation of the network accompanied by evaporation of methanol and water. A sharp exothermic peak around $200{ }^{\circ} \mathrm{C}$ derived from the combustion of P123 is observed in the gel phase while no peaks are observed in the solvent phase. Namely, it is confirmed that the most of P123 is distributed not in the solvent phase but in the gel phase, due to the too high hydrophobicity (high PO/EO ratio). As a result, the phase separation tendency is not suppressed but rather accelerated, and the MSQ networks form less homogeneous structure in the longer length scale. On the other hand, only a small exothermic peak can be seen in the unwashed gel phase in the case of F127, 
and no evidence of F127 is observed in the washed sample (not shown). From these results, we can propose that the F127 molecules are not embedded in the MSQ networks, but are weakly adsorbed at the solvent-MSQ interfaces by hydrophobic interaction. Highly polar solvent phase and less polar gel phase accommodate F127 (high HLB) and P123 (low HLB), respectively in each system.

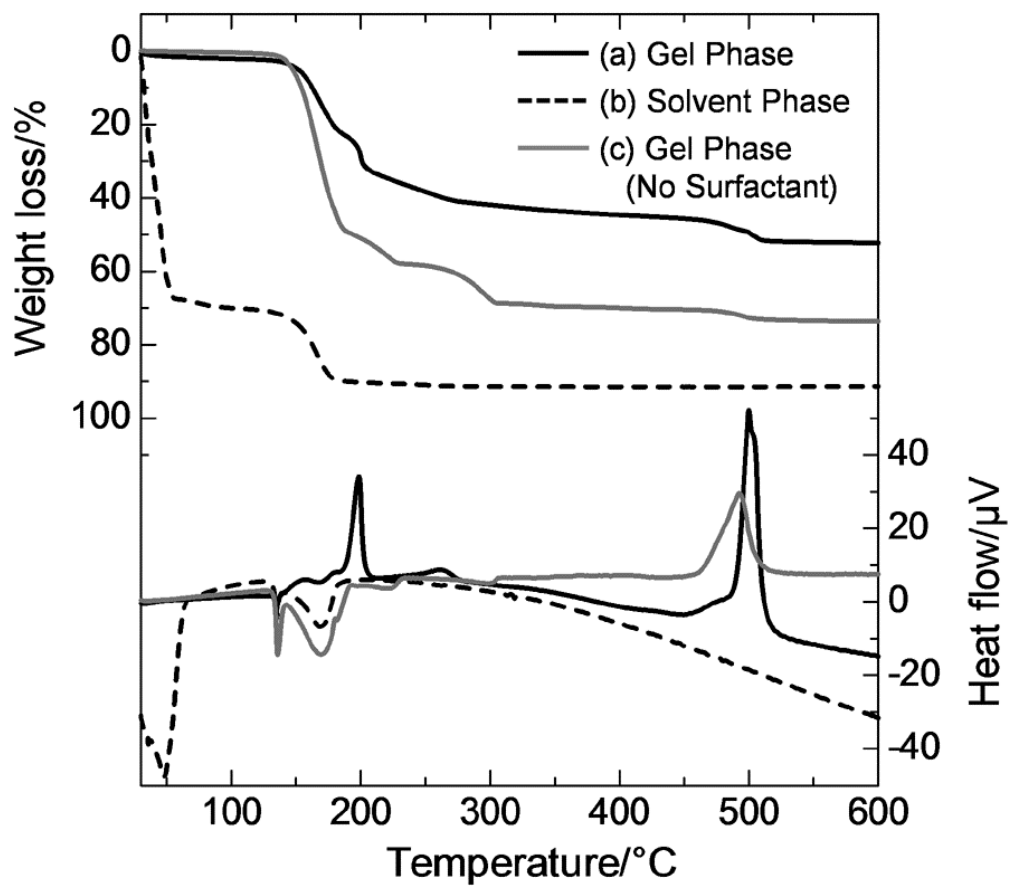

Fig. 7 TG-DTA curves for a macroscopically phase-separated sample prepared with P123; (a) gel phase (precipitate), (b) solvent phase (supernatant), and (c) gel phase of a sample prepared without surfactant.

From all these observations, it can be summarized that the relative distribution of MSQ and surfactant gives the critical influence on the suppression of phase separation, and the distribution is predominantly governed by the HLB (or PO/EO ratio) of each surfactant. The MSQ networks must be rendered hydrophilic for the suppression of phase separation, and it can be achieved by adsorbing surfactant at the MSQ-solvent interfaces through hydrophobic interaction. Surfactant should be moderately hydrophobic for avoiding absorption and enhancing effective adsorption, while at the same time reasonable EO chains are required to ensure the ability to make the MSQ network hydrophilic. To fulfill 
these complex requirements, we found surfactant P105 is the most effective one to obtain transparent aerogels. Surfactants F127, F108 and F68 are also effective for the same purpose. In the following section we further discuss on these issues with NMR data.

\section{NMR studies}

Fig. 8 shows in-situ ${ }^{1} \mathrm{H}$ NMR spectra which were taken on the typical starting composition (1.0 $\mathrm{g}$ of F127, $3.0 \mathrm{~g}$ of urea, $10 \mathrm{~mL}$ of $5 \mathrm{mM}$ HOAc solution in $\mathrm{D}_{2} \mathrm{O}$. and $5 \mathrm{~mL}$ of MTMS) sample polymerized at $60{ }^{\circ} \mathrm{C}$ to elucidate the MSQ-surfactant interaction mechanism. The ${ }^{1} \mathrm{H}$ signals related to the organic groups are assigned according to previous reports ${ }^{26,40}$ and an extra experiment has also been done on the system without surfactant (not shown). All of the spectra moved toward the lower magnetic field with reaction time, since $\mathrm{pH}$ in the solution increases by hydrolysis of urea. The peaks derived from methyl protons at $0-0.2$ ppm are assigned to those bonded with $\mathrm{T}^{0}$ (higher field), $\mathrm{T}^{2}$ and $\mathrm{T}^{3}$ (lower), and $\mathrm{T}^{1}$ (middle) silicon species of MTMS-derived networks. These peaks increase in the lower magnetic field side and decrease in the higher field as condensation progresses because less electron-withdrawing $-\mathrm{OCH}_{3}$ groups are replaced with more electron-withdrawing $-\mathrm{OH}$, -OSi groups as the reaction proceeds, which reduces the electron density on the methyl protons. 

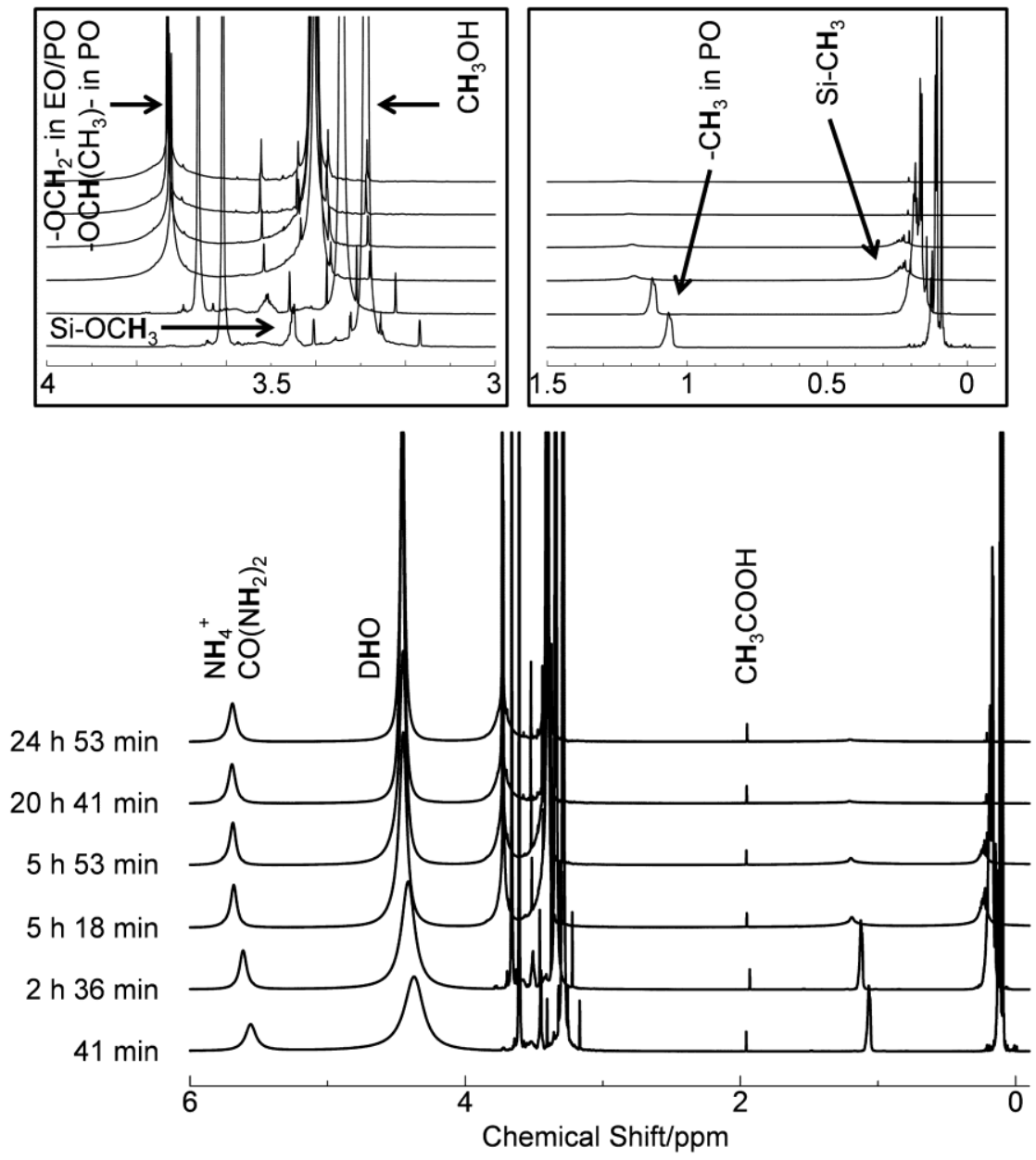

Fig. 8 Time-resolved ${ }^{1} \mathrm{H}$ NMR spectra of MTMS-F127-D $2 \mathrm{O}-$ Urea-Acetic acid system at $333 \mathrm{~K}$ obtained at different times after the hydrolysis for $30 \mathrm{~min}$ (insets are the enlarged spectra).

In this figure, the peaks related to methyl groups in the MSQ networks $(\sim 0.2 \mathrm{ppm})$ and PO units $(\sim 1.1 \mathrm{ppm})$ become simultaneously and significantly broadened in the third spectrum ( $5 \mathrm{~h} 18 \mathrm{~min}$ ). It is plausible that gelation occurred between $2 \mathrm{~h} 36 \mathrm{~min}$ and $5 \mathrm{~h}$ 18 min, which make the mobility of methyl groups in MSQ networks significantly decreased. While the peak related to $-\mathrm{OCH}_{2}-$ in $\mathrm{EO}$ and $\mathrm{PO}$ and $-\mathrm{OCH}_{2} \mathrm{CH}\left(\mathrm{CH}_{3}\right)^{-}$in PO near 3.6 ppm are only slightly decreased and broadened, still sharp peaks can be seen in the figure compared to the methyl groups in MSQ networks and PO units mentioned above. This shows that most of the EO units are not tightly restrained near the gel networks by 
hydrogen bonding during and after the gelation. That is, EO units are away from the MSQ networks and exposed toward the solvent phase while a large part of PO units exist near the MSQ networks presumably through hydrophobic interaction when becoming close to the gel point. ${ }^{33-35}$ The interaction mechanism between MTMS-derived condensates and triblock copolymers therefore changes from hydrogen bonding between silanol in the networks and ether oxygen in EO parts, to hydrophobic interaction between MSQ and PO parts with the progress of polycondensation. This mechanism agrees with the general trend that MTMS-derived condensates are relatively hydrophilic due to the abundant silanol groups in the earlier stage of polycondensation, and become increasingly hydrophobic because silanol groups are consumed to form siloxane bonds in the later stage.

Fig. 9 shows solid-state ${ }^{29} \mathrm{Si} \mathrm{CP} / \mathrm{MAS}$ NMR spectra of the samples prepared with different amounts of F127. It can be seen from this figure that $\mathrm{T}^{2}$ species decrease, i.e. crosslinking density of the gels increases, with increasing amount of surfactant. The $\mathrm{T}^{3} / \mathrm{T}^{2}$ area ratios are, respectively, 3.36, 4.54, 5.67, and 5.70 with increasing amount of F127. Since the macroscopic phase separation tendency of hydrophobic MSQ networks from the solvent becomes higher with decreasing F127, the networks are segregated from the solution in the earlier stage of polycondensation. Therefore the more hydrogen bonding should remain at the onset of phase separation, which decreases the crosslinking density in the resulting gels. With the higher amount of surfactant, compatibility between MSQ oligomers and polar solvent becomes higher, which allows more enhanced condensation of MSQ and rather compact colloidal form of condensates would result. At the same time, the porous structures are formed presumably through microphase separation of MSQ condensates from F127-rich solution. ${ }^{23}$ Therefore the addition of surfactant not only suppresses macroscopic phase separation but increases crosslinking density by enhancing the polycondensation. However, with an excess of F127 (or other surfactants except for L35 and P123), the resultant networks consist of entanglement of compact MSQ networks and abundant surfactant, which leads to swelling with alcoholic solvents as discussed above. 


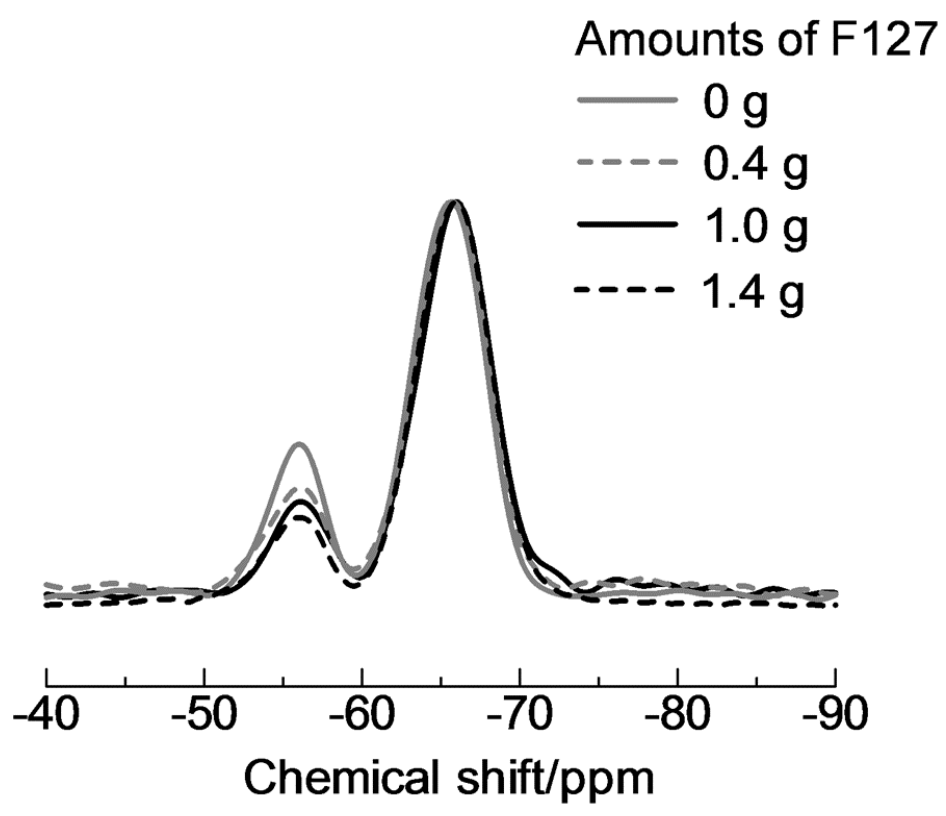

Fig. 9 Solid-state ${ }^{29} \mathrm{Si}$ CP/MAS NMR spectra of the samples prepared with different amounts of F127. These spectra are normalized with respect to the peak height of $\mathrm{T}^{3}$ (at $-66 \mathrm{ppm})$.

\section{Conclusions}

Changes of properties of MSQ aerogels are systematically investigated with different concentrations of triblock copolymer surfactant in the two-step sol-gel system of MTMS. Nonionic PEO- $b$-PPO- $b$-PEO triblock copolymers with different molecular weights and $\mathrm{PO} / \mathrm{EO}$ ratios (or HLB value) are employed to investigate the effects on the phase separation behavior of the MTMS-derived networks from polar solvent. It is found that both molecular weight and PO/EO ratio give an important influence on the suppression of phase separation. Surfactants with high molecular weight and moderate PO/EO ratio, i.e. such as Pluronic P105, F127, F108 and F68, can effectively suppress phase separation because these molecules adsorb on the hydrophobic MSQ networks through hydrophobic interaction between MSQ and PO units with hydrophilic EO units exposed to the polar solvent. Transparent aerogels cannot be obtained from the surfactants which have too low molecular weight (L35) or too high hydrophobicity (P123). The short surfactant cannot effectively interact with MSQ networks and too hydrophobic surfactant is incorporated into the MSQ networks, both of which give less influence on the hydrophobicity of condensates. 
Liquid-state in-situ ${ }^{1} \mathrm{H}$ NMR measurement shows that the PO units of surfactant lose mobility on gelation, which suggests that PO units are constrained near the MSQ networks through hydrophobic interaction. The MSQ condensates are rather hydrophilic at the earlier stage of polycondensation (far before gelation), during which hydrogen bonding between silanols in condensates and EO units presumably dominates in analogy with the silica systems. However, at the later polycondensation stage when the MSQ condensates have consumed most of the silanols, hydrophobic interaction becomes predominant in turn. It is also found from the solid-state NMR results that the presence of higher concentrations of triblock copolymer increases crosslinking density of MSQ networks in addition to the suppression of phase separation.

Through understanding the role of surfactant in the MTMS-derived sol-gel system, a better design of low-density and transparent MSQ aerogels has now become possible, which will open the way to more extended applications of these unique porous materials.

\section{Acknowledgements}

Technician Ms. Kyoko Ohmine at Institute for Chemical Research, Kyoto University is highly acknowledged for the measurements of liquid state NMR. The present work was financially supported by Advanced Low Carbon Technology Research and Development Program (ALCA) from Japan Science and Technology Agency (JST). Also acknowledged is the Grant-in-Aid for Scientific Research (Scientific Research (c) No. 24550253 for K.K.) from the Ministry of Education, Culture, Sports, Science and Technology (MEXT), Japan.

\section{Notes and references}

${ }^{a}$ Department of Chemistry, Graduate School of Science, Kyoto University, Kitashirakawa, Sakyo-ku, Kyoto 606-8502, Japan. Fax: +81-75-753-7673; Tel: +81-75-753-7673; E-mail: kanamori@kuchem.kyoto-u.ac.jp

${ }^{b}$ Institute for Chemical Research, Kyoto University, Uji, Kyoto 611-0011, Japan. Fax: +81-774-38-3149; Tel: +81-774-38-3149; E-mail: kaji@scl.kyoto-u.ac.jp

$\dagger$ Electronic Supplementary Information (ESI) available: [details of any supplementary information available should be included here]. See DOI: 10.1039/b000000x/

1 G. J. A. A. Soler-Illia, C. Sanchez, B. Lebeau and J. Patarin, Chem. Rev., 2002, 102, 4093-4138.

2 Q. Huo, D. I. Margolese, U. Ciesla, D. G. Demuth, P. Feng, T. E. Gier, P. Sieger, A. 
Firouzi, B. F. Chmelka, F. Schüth and G. D. Stucky, Chem. Mater., 1994, 6, 1176-1191.

3 J. Frasch, B. Lebeau, M. Soulard and J. Patarin, Langmuir, 2000, 16, 9049-9057.

4 D. Zhao, Q. Huo, J. Feng, B. F. Chmelka and G. D. Stucky, J. Am. Chem. Soc., 1998, 120, 6024-6036.

5 J. Fricke and T. Tillotson, Thin Solid Films, 1997, 297, 212-223.

6 N. Hüsing and U. Schubert, Angew. Chem. Int. Ed., 1998, 37, 22-45.

7 A. C. Pierre and G. M Pajonk, Chem. Rev., 2002, 102, 4243-4265.

8 S. S. Kistler, Nature, 1931, 127, 741.

9 G. M. Pajonk, App. Catal., 1991, 72, 217-266.

10 L. W. Hrubesh, J. Non-Cryst. Solids, 1998, 225, 335-342.

11 S. Hæreid, J. Anderson, M. A. Einarsrud, D. W. Hua and D. M. Smith, J. Non-Cryst. Solids, 1995, 185, 221-226.

12 M.-A. Einarsrud, E. Nilsen, A. Rigacci, G. M. Pajonk, S. Buathier, D. Valette, M. Durant, B. Chevalier, P. Nitz and F. Ehburger-Doll, J. Non-Cryst. Solids, 2001, 285, 1-7.

13 G. Reichenauer, J. Non-Cryst. Solids, 2004, 350, 189-195.

14 B. M. Novak, D. Auerbach and C. Verrier, Chem. Mater., 1994, 6, 282-286.

15 N. Leventis, C. Sotiriou-Leventis, G. Zhang and A.-M. M. Rawashdeh, Nano Lett., 2002, 2, 957-960.

16 M. A. B. Meador, E. F. Fabrizio, F. Ilhan, A. Dass, G. Zhang, P. Vassilaras, J. C. Johnston and N. Leventis, Chem. Mater, 2005, 17, 1085-1098.

17 N. Hüsing and U. Schubert, J. Sol-Gel Sci. Technol., 1997, 8, 807-812.

18 H. E. Rassy and A. C. Pierre, J. Non-Cryst. Solids, 2005, 351, 1603-1610.

19 A. V. Rao, S. D. Bhagat, H. Hirashima and G. M. Pajonk, J. Colloid Interface Sci., 2006, 300, 279-285.

20 K. Kanamori, M. Aizawa, K. Nakanishi and T. Hanada, Adv. Mater., 2007, 19, 1589-1593.

21 K. Kanamori, M. Aizawa, K. Nakanishi and T. Hanada, J. Sol-Gel. Sci. Technol., 2008, 48, 172-181.

22 K. Kanamori, K. Nakanishi and T. Hanada, J. Ceram. Soc. Jpn., 2009, 117, 1333-1338.

23 K. Kanamori, Y. Kodera, G. Hayase, K. Nakanishi and T. Hanada, J. Colloid Interface Sci., 2011, 357, 336-344.

24 K. Kanamori and K. Nakanishi, Chem. Soc. Rev., 2011, 40, 754-770.

25 N. Baccile, G. Laurent, C. Bonhomme, P. Innocenzi and F. Babonneau, Chem. Mater., 
2007, 19, 1343-1354.

26 N. A. Melosh, P. Lipic, F. S. Bates, F. Wudl, G. D. Stucky, G. H. Fredrickson and B. F. Chmelka, Macromolecules, 1999, 32, 4332-4342.

27 P. Alexandridis and T. A. Hatton, Colloids Surf., A, 1995, 96, 1-46.

28 Pluronic and Tetronic Surfactants. Technical Brochure; BASF Corp.: Parsippany, NJ, 1989.

29 K. Takeda, Rev. Sci. Instrum., 2007, 78, 033103.

30 K. Takeda, J. Magn. Reson., 2008, 192, 218-229.

31 M. Malmsten, P. Linse and T. Cosgrove, Macromolecules, 1992, 25, 2474-2481.

32 D. Santhiya, G. Nandini, S. Subramanian, K. A. Natarajan andS. G. Malghan, Colloids Surf., A, 1998, 133, 157-163.

33 L. M. A. van de Steeg and C.-G. Gölander, Colloids Surf., 1991, 55, 105-119.

34 C. G. P. H. Schroén, M. A. Cohen Stuart, K. van der Voort Maarschalk, A. van der Padt and K. van't Riet, Langmuir, 1995, 11, 3068-3074.

35 R. J. Green, S. Tasker, J. Davies, M. C. Davies, C. J. Roberts and S. J. B. Tendler, Langmuir, 1997, 13, 6510-6515.

36 J. A. Shar, T. M. Obey, T. Cosgrove, Colloids Surf., A, 1998, 136, 21-33.

37 S. Yang, P. A. Mirau, C.-S. Pai, O. Nalamasu, E. Reichmanis, E. K. Lin, H.-J. Lee, D. W. Gidley and J. Sun, Chem. Mater., 2001, 13, 2762-2764.

38 P. A. Mirau and S. Yang, Chem. Mater., 2002, 14, 249-255.

39 P. M. Schaber, J. Colson, S. Higgins, D. Thielen, B. Anspach and J. Brauer, Thermochim. Acta, 2004, 424, 131-142.

40 B. Alonso, F. Fayon, D. Massiot, H. Amenitsch, L. Malfatti, T. Kidchob, S. Costacurta and P. Innocenzi, J. Phys. Chem. C, 2010, 114, 11730-11740. 